\title{
A Novel Way of Standardization of ICG Lymphangiography Reporting
}

\author{
Ashok Basur Chandrappa ${ }^{1}$ Ritu Batth ${ }^{1} \quad$ Srikanth Vasudevan ${ }^{1} \quad$ Anantheswar Yellambalase N ${ }^{1}$ \\ Dinkar Sreekumar ${ }^{1}$ \\ ${ }^{1}$ Department of Plastic and Reconstructive Surgery, Manipal \\ Hospital, Bangalore, India \\ Address for correspondence Ritu Batth, DNB, Department \\ of Plastic Surgery, Manipal Hospital, Bangalore, 560017, India \\ (e-mail: dr.ritubatth@gmail.com).
}

Indian J Plast Surg:2020;53:377-380

\begin{abstract}
Background Indocyanine green (ICG) lymphangiography is being increasingly employed to assess the severity of lymphedema, locate the areas of patent linear lymphatics and dermal backflow and plan treatment. This study suggests a novel method of reporting ICG findings in extremities to enable easy understanding among surgeons and physiotherapists and avoid repeat testing when a patient visits a disparate lymphedema center or clinician.

Methods A reporting protocol was developed in the lymphedema clinic of the plastic surgery department, and patients were asked to bring along the report in every subsequent review. The ICG findings were recorded on the fluorescence imaging system as well. The report was prepared by one and analyzed by two different clinicians without

Keywords

- ICG reporting

- lymphedema

- standardization repeating the test on 10 consecutive patients.

Results The interrater reliability of findings in the report was found to be $98.7 \%$ among the three clinicians.

Conclusion The reporting system was found to be illustratable and reproducible
\end{abstract}

\section{Introduction}

Lymphedema is being increasingly dealt by reconstructive and microsurgeons in the wake of increasing surgical treatment modalities. The plan of management depends on factors like level of subcutaneous fibrosis, associated skin changes, degree of increase in limb girth and, most importantly, availability of patent lymphatics or extent of channel destruction. Intradermal injection of indocyanine green (ICG) dye, followed by infrared scan, is a portable, quick and safe way of delineating the lymphatics, which does not employ any radioactive exposure. The findings can be recorded and shared among any number of clinicians. Although lymphedema can be classified universally, according to ICG findings, the system of reporting the findings is very exhaustive, nonspecific and not easily reproducible ( - Fig. 1 ). This study proposes a reporting system for precisely locating different representations of the dye in the lymphatics, thereby minimizing uncertainty.

published online

November 19, 2020
DOI https://doi.org/

10.1055/s-0040-1716436 ISSN 0970-0358.

\section{Materials and Methods}

The patient was asked to change into a hospital gown and placed in a dark room. The Irillic.nm flourescence imaging system (Irillic, India) was prepared and camera kept on a standby mode. Injection sites were sterilized with betadine solution. For upper limb, first and fourth web space, radial and ulnar aspect of the volar wrist were chosen as injection sites, whereas for lower limb, first web space and lateral to tendoachilles were injected based on cadaveric lymphatic studies. ${ }^{1,2}$ Additional injections near the elbow or knee can be given to hasten the proximal dye uptake, and in severe lymphedema, with diffuse dermal backflow distally. An amount of $0.5 \mathrm{~mL}$ of $2 \%$ lignocaine was injected, followed by $0.5 \mathrm{~mL}$ of Aurogreen dye (Aurolab, India) (ICG-25 mg vial diluted with $10 \mathrm{~mL}$ distilled water) injected intradermally into each site, using a $1 \mathrm{~mL}$ syringe with $31 \mathrm{G}$ needle. The patient was asked to walk around, massage manually and open or close a fist repeatedly. The scan was repeated every 15 minutes till the

(C2020. Association of Plastic Surgeons of India.

This is an open access article published by Thieme under the terms of the Creative Commons Attribution-NonDerivative-NonCommercial-License, permitting copying and reproduction so long as the original work is given appropriate credit. Contents may not be used for commercial purposes, or adapted, remixed, transformed or built upon. (https://creativecommons.org/licenses/by-nc-nd/4.0/).

Thieme Medical and Scientific Publishers Pvt. Ltd. A-12, 2nd Floor, Sector 2, Noida-201301 UP, India 

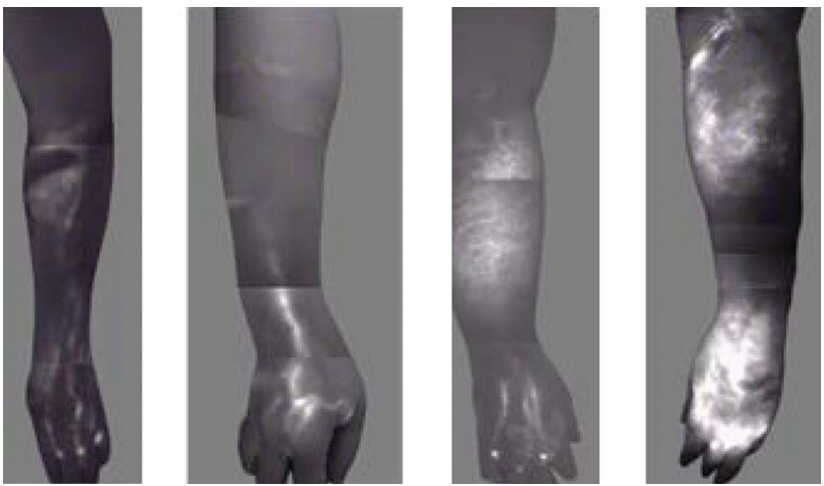

Fig. 1 Lymphedema classification based on ICG lymphangiography (Source: Chang DW, Suami H, Skoracki R. A prospective analysis of 100 consecutive lymphovenous bypass cases for treatment of extremity lymphedema. Plast Reconstr Surg 2013; 132:1305-14).



Fig. 2 Linear lymphatics.

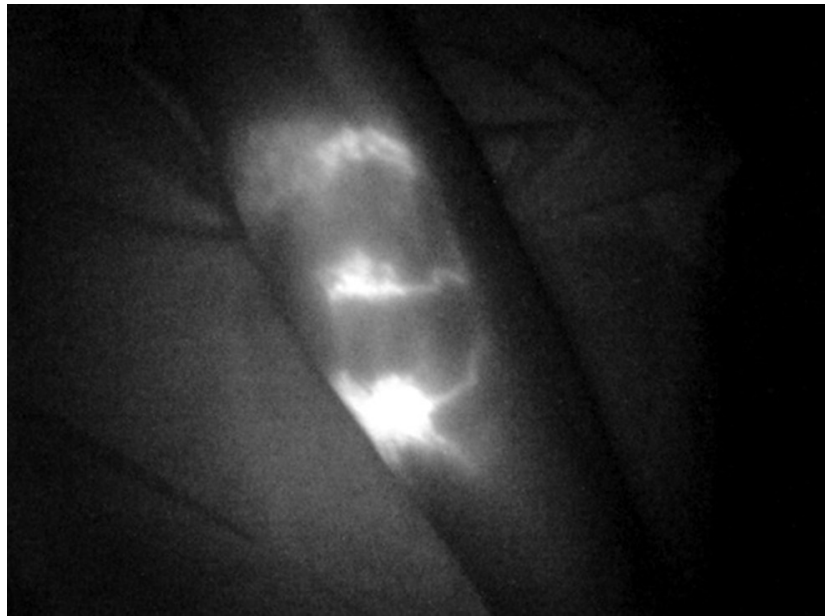

Fig. 3 Splash pattern of dermal backflow.

dye reached axilla or groin or dye showed no progress for 45 minutes.

The report included the following findings:

1. Linear lymphatics (-Fig. 2).

2. Splash pattern of dermal backflow (-Fig. 3 ).

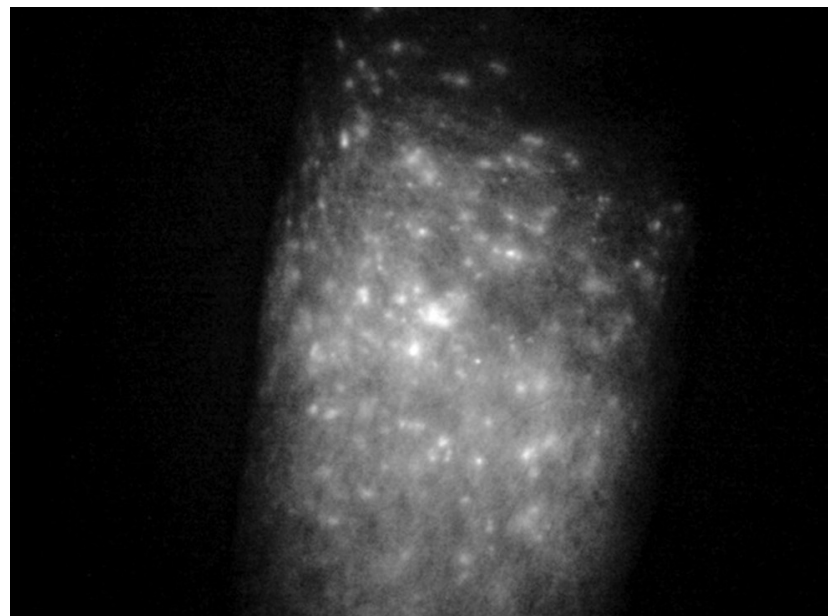

Fig. 4 Stardust pattern of dermal backflow.

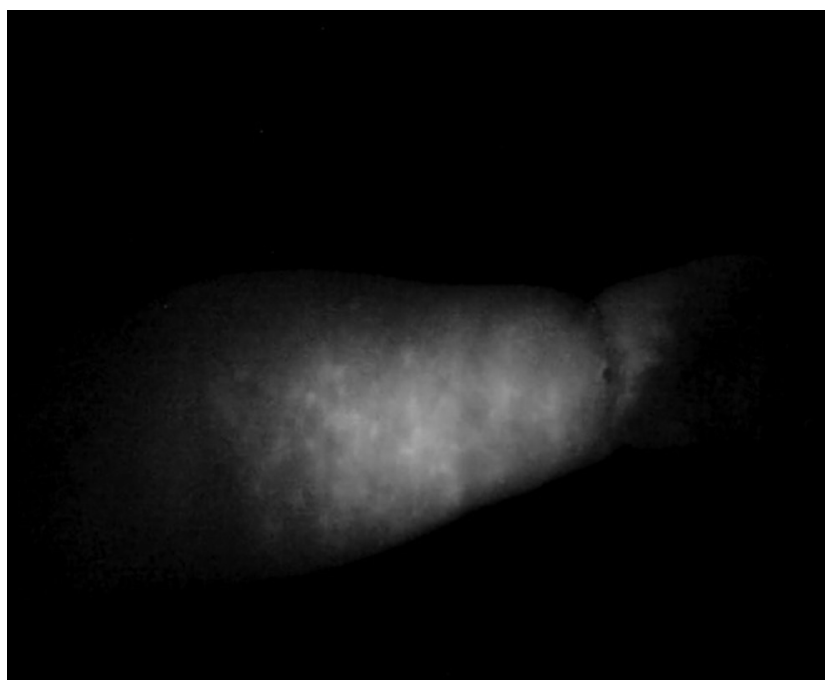

Fig. 5 Diffuse pattern of dermal backflow.

3. Stardust pattern of dermal backflow ( - Fig. 4).

4. Diffuse pattern of dermal backflow (-Fig. 5 ).

5. Blank zone.

A four-component code was created for each of the above findings as depicted below.

1. Right/Left-R/L

2. Flexor/Extensor-F/E

3. Zone/Wrist/Ankle-Z/W/A ( - Table 1) (-Fig. 6)

4. Radial/Ulnar/Medial/Lateral-R/U/M/L

For instance, linear lymphatics on flexor aspect of the right forearm in zone 3 , that is, within 8 to $12 \mathrm{~cm}$ from the wrist along the radial aspect was reported as Linear lymphatics-"RFZ3R."

Splash pattern on extensor aspect of the left leg in zone 7 and 8 , that is, within 24 to $32 \mathrm{~cm}$ from the ankle along the medial aspect was reported as Splash-"LEZ7+8M" (-Table 2).

The findings were charted on a reporting sheet prepared by the team, illustrating the patient identification details, relevant history, volumetric findings, proposed reporting format, and graphic representation of the ICG findings ( - Fig. 7). 
Table 1 Description of the limb zones

\begin{tabular}{|l|l|}
\hline Zone/wrist/ankle & Distance from wrist/ankle $(\mathbf{c m})$ \\
\hline Midpalm/midfoot & Midpalm/midfoot \\
\hline W/A & 0 \\
\hline Z1 & 4 \\
\hline Z2 & 8 \\
\hline Z3 & 12 \\
\hline Z4 & 16 \\
\hline Z5 & 20 \\
\hline Z6 & 24 \\
\hline Z7 & 28 \\
\hline Z8 & 32 \\
\hline Z9 & 36 \\
\hline Z10 & 40 \\
\hline Z11 & 44 \\
\hline Z12 & 48 \\
\hline Z13 & 52 \\
\hline Z14 & 56 \\
\hline
\end{tabular}

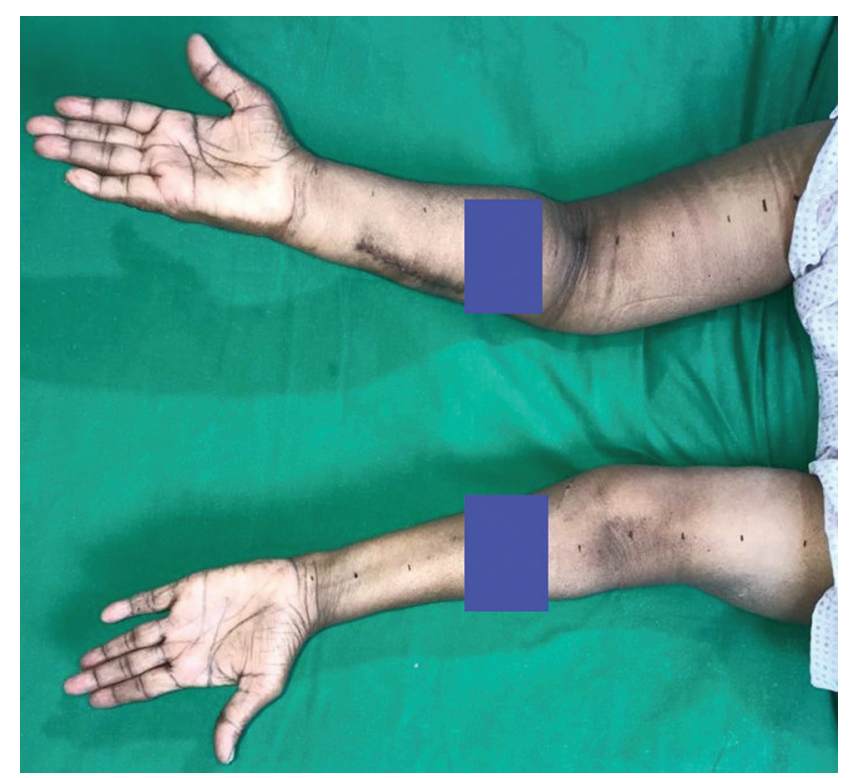

Fig. 6 Marking the zones in upper limbs.

The same report was interpreted by two different clinicians, without any aid from the primary clinician, on a series of 10 patients, and interrater reliability was calculated using the following formula:

$$
\text { Interrater reliability }=\frac{\text { Total no. of correct tests }}{\text { Total no. of tests }} \times 100
$$

\section{Results}

The interrater reliability with which the ICG findings could be interpreted and charted among the three clinicians was $98.7 \%$.
Table 2 The proposed four-component reporting code

\begin{tabular}{|l|l|l|l|l|}
\hline Finding & $\begin{array}{l}\text { Right/ } \\
\text { Left }\end{array}$ & $\begin{array}{l}\text { Flexor/ } \\
\text { Extensor } \\
\text { surface }\end{array}$ & Zone & Border \\
\hline $\begin{array}{l}\text { Linear } \\
\text { lymphatics }\end{array}$ & R/L & F/E & Zx & R/U/M/L \\
\hline Splash & & & & \\
\hline Stardust & & & & \\
\hline Diffuse & & & & \\
\hline Blank & & & & \\
\hline
\end{tabular}

$\mathrm{L}=$ lateral; $\mathrm{M}=$ medial; $\mathrm{R}=$ radial; $\mathrm{U}=$ ulnar.

\section{Discussion}

Several methods have been reported to detect lymphatic channels, for example, including magnetic resonance imaging, computed tomography, ultrasonography, lymphoscintigraphy and ICG lymphangiography. ${ }^{3-8}$ ICG is a green fluorescent dye with no radioactive potential, which travels fast in the body, being a water soluble preparation. Up to $2 \mathrm{~cm}$ deep lymphatics can be visualized and assessed by ICG lymphangiography, based on the penetration level of near infrared rays. ${ }^{9}$ The camera handpiece consists of an excitation light source with a wavelength of $770 \mathrm{~nm}$ and a near infrared detector that filters and collects the fluorescence signals above $800 \mathrm{~nm}$. When ICG is excited by the light source, the emitted fluorescence is captured and displayed in real-time using detector and a custom software. These fluorescence signals can be stored as images and videos and reviewed later. ICG lymphangiography findings include either fluorescent linear lymphatic channels or dermal backflow. Linear channels represent the normal functional superficial lymphatics. Dermal backflow is a pathological finding which presents as different patterns, as per the severity of damage. Splash pattern represents an early stage of valve destruction, with scattered dye and tortuous lymphatic channels. Stardust or milky way pattern is an indicator of progression with diffuse illuminated background and scattered bright fluorescent spots. Diffuse pattern of dermal backflow indicates a severe advanced stage of lymphedema with wall thickening and lumen stenosis. It is seen as a widespread fluorescence with no areas of bright spots. ${ }^{2}$ These patterns map the areas with available lymphatics as well as areas of destruction, thereby dictating the surgical interventions feasible. Blank zone in proximal extremities indicates poor dye uptake and thus can either be interpreted as a higher grade of lymphedema or supplemented with additional proximal dye injections, in order to outline the backflow pattern in these zones. Despite being a patient and clinician friendly investigation, the interpretation of findings following ICG lymphangiography is time consuming and lacks standardization. The disorderly system of reporting makes it arduous to locate and mark the exact location and extent of linear channels and dermal backflow on any future follow-up unless the recorded findings are available. This can lead to needless repetition of the test when the patients seek 




Fig. 7 The proposed reporting system sheet.

a different clinician or center or the same clinician encounters the patient after a routine follow-up of months. Likewise, the report can also facilitate better communication and coordination among the surgeons and physiotherapists regarding the type and intensity of physiotherapy needed and in outlining common treatment goals. Like the standardization of classification of lymphedema has been in practice and enables better understanding of the severity and progression of the disease, the findings of the lymphography, if standardized, can ensure a quick and systematic management of lymphedema patients. This does not substitute on table ICG marking but helps in preoperative patient counselling with reference to the need for surgery, planning the type of surgery, guiding the physiotherapist in decongestive physiotherapy, comparing the progress of the disease, and monitoring postoperative progress in case the old ICG recordings are not available. As the ICG test is a dynamic investigation whose results vary with time, this reporting system is aimed at avoiding unplanned repeat studies before the stipulated time due to lack of reliable information about the previous dye study. As depicted by this study, the extent of interpretation of an ICG scan reported using the proposed symbolic representation system among multiple clinicians was found to be reliable and obviated the need for a repeat scan, thereby empowering consistency to a clinician's assimilation.

\section{Financial Disclosures}

None.

\section{Earlier Presentation}

None.

\section{Conflicts of Interest}

None declared.

\section{Acknowledgments}

None.

\section{References}

1 Suami H, Heydon-White A, Mackie H, Czerniec S, Koelmeyer L, Boyages J. A new indocyanine green fluorescence lymphography protocol for identification of the lymphatic drainage pathway for patients with breast cancer-related lymphoedema. BMC Cancer 2019;19(1):985

2 Narushima M, Yamamoto T, Ogata F, Yoshimatsu H, Mihara M, Koshima I. Indocyanine green lymphography findings in limb lymphedema. J Reconstr Microsurg 2016;32(1):72-79

3 Henze E, Schelbert HR, Collins JD, Najafi A, Barrio JR, Bennett LR. Lymphoscintigraphy with Tc-99m-labeled dextran. J Nucl Med 1982;23(10):923-929

4 Szuba A, Shin WS, Strauss HW, Rockson S. The third circulation: radionuclide lymphoscintigraphy in the evaluation of lymphedema. J Nucl Med 2003;44(1):43-57

5 Tomczak H, Nyka W, Lass P. Lymphoedema: lymphoscintigraphy versus other diagnostic techniques-a clinician's point of view. Nucl Med Rev Cent East Eur 2005;8(1):37-43

6 Case TC, Witte CL, Witte MH, Unger EC, Williams WH. Magnetic resonance imaging in human lymphedema: comparison with lymphangioscintigraphy. Magn Reson Imaging 1992;10(4): 549-558

7 Gamba JL, Silverman PM, Ling D, Dunnick NR, Korobkin M. Primary lower extremity lymphedema: CT diagnosis. Radiology 983;149(1):218

8 Doldi SB, Lattuada E, Zappa MA, Pieri G, Favara A, Micheletto G. Ultrasonography of extremity lymphedema. Lymphology 1992;25(3):129-133

9 Unno N, Nishiyama M, Suzuki M, et al. Quantitative lymph imaging for assessment of lymph function using indocyanine green fluorescence lymphography. Eur J Vasc Endovasc Surg 2008;36(2):230-236 